\title{
Most Colorful Example of Genetic Assimilation? Exploring the Evolutionary Destiny of Recurrent Phenotypic Accommodation
}

\author{
Alexander V. Badyaev, Ahva L. Potticary, and Erin S. Morrison \\ Department of Ecology and Evolutionary Biology, University of Arizona, Tucson, Arizona 85721 \\ Submitted August 12, 2016; Accepted March 3, 2017; Electronically published May 15, 2017 \\ Online enhancements: appendix.
}

ABSTRACT: Evolution of adaptation requires both generation of novel phenotypic variation and retention of a locally beneficial subset of this variation. Such retention can be facilitated by genetic assimilation, the accumulation of genetic and molecular mechanisms that stabilize induced phenotypes and assume progressively greater control over their reliable production. A particularly strong inference into genetic assimilation as an evolutionary process requires a system where it is possible to directly evaluate the extent to which an induced phenotype is progressively incorporated into preexisting developmental pathways. Evolution of diet-dependent pigmentation in birds - where external carotenoids are coopted into internal metabolism to a variable degree before being integrated with a feather's developmental processes - provides such an opportunity. Here we combine a metabolic network view of carotenoid evolution with detailed empirical study of feather modifications to show that the effect of physical properties of carotenoids on feather structure depends on their metabolic modification, their environmental recurrence, and biochemical redundancy, as predicted by the genetic assimilation hypothesis. Metabolized carotenoids caused less stochastic variation in feather structure and were more closely integrated with feather growth than were dietary carotenoids of the same molecular weight. These patterns were driven by the recurrence of organism-carotenoid associations: commonly used dietary carotenoids and biochemically redundant derived carotenoids caused less stochastic variation in feather structure than did rarely used or biochemically unique compounds. We discuss implications of genetic assimilation processes for the evolutionary diversification of diet-dependent animal coloration.

Keywords: genetic assimilation, developmental plasticity, phenotypic accommodation, carotenoids.

\footnotetext{
* Corresponding author; e-mail: abadyaev@email.arizona.edu. ORCIDs: Potticary, http://orcid.org/0000-0002-1157-5315; Morrison, http:// orcid.org/0000-0002-4487-6915.
}

Am. Nat. 2017. Vol. 190, pp. 266-280. (C) 2017 by The University of Chicago. 0003-0147/2017/19002-57175\$15.00. All rights reserved. DOI: $10.1086 / 692327$

\author{
Introduction \\ Genetic Assimilation as a Case of Upstream \\ Traversing of a Developmental Hierarchy
}

Adaptive microevolutionary modifications usually arise at the periphery of developmental hierarchies, where they affect fewer subsequent modifications and where the external environment provides regulation and a context for their expression. Yet the evolutionary retention of these modifications is greater at upstream developmental stages, where their determination and inheritance are less dependent on specific environmental contexts and are under greater organismal control (reviewed in Wilkins 2001; Davidson 2006; Peter and Davidson 2015). Although outcomes of both upstream and downstream shifts (hereafter traversing) of controls along developmental hierarchies are well established in macroevolution and development (King et al. 2008; Newman 2012; Buitrago-Delgado et al 2015; Levin et al. 2016), it is challenging to study it as an ongoing process in a microevolutionary context.

The genetic assimilation phenomenon - where adaptive change induced by the environment becomes stabilized by internal organismal processes - might be viewed as a case of upstream traversing of developmental hierarchies by controls of induced phenotypes (Baldwin 1902; Schmalhausen 1938; West-Eberhard 2003). Indeed, classical introductions of the genetic assimilation phenomenon argued that such a developmental shift of regulators enables incorporation of novel environmental input without disrupting homeostasis of already present structures and thus should be a dominant mode of microevolution in complex multicellular organisms (Cope 1887; Baldwin 1896; Osborn 1897; Chetverikov 1926; Schmalhausen 1938; Gause 1940; Waddington 1941). This is because such an upstream shift interferes with fewer downstream regulators and is therefore less disruptive, can accomplish the spread of adaptive phenotypes on ecologically relevant timescales and across 
many individuals, and capitalizes on a greater redundancy of phenotypic, physiological, and genomic networks of terminal developmental states. Indeed, several studies have documented or inferred an upstream shift in the distribution of genetic or genomic controls between ancestral and derived lineages and interpreted this shift as evidence for genetic assimilation (Heil et al. 2004; Wund et al. 2008; Emera et al. 2012; Diggle and Miller 2013; Schlichting and Wund 2014). However, variable positions of determinants of a trait along ontogenies might be an inevitable consequence of the recurrent use and reshuffling of ancient genes for contemporary functions (West-Eberhard 2005; Badyaev 2014), such that the shift itself and associated heterochronic and heterotopic phenotypic effects are ubiquitous and thus insufficient for proving genetic assimilation.

\section{Establishing Genetic Assimilation as an Evolutionary Mechanism}

The establishment of genetic assimilation as a mechanism behind developmental change requires its study as a process, because once established, the patterns of morphological variation due to upstream and downstream traversing of developmental hierarchies are indistinguishable. Further, because genetic assimilation is a transient (Pigliucci and Murren 2003) and rapid evolutionary process (Waddington 1959; Rutherford and Lindquist 1998; Suzuki and Nijhout 2006), documenting that extant forms likely had environmentally induced ancestral states offers only indirect inference because both are endpoint evolutionary solutions.

Proximately, the process of genetic assimilation refers to internalization of developmental controls of an adaptive modification, such as when trait expression lessens its dependence on the environment and different mechanisms regulate expression of the same trait across evolutionary stages in a lineage (Gause 1940; Waddington 1953; Schlichting and Pigliucci 1998; Pigliucci et al. 2006; Badyaev and Oh 2008; Schlichting and Wund 2014; Sikkink et al. 2014; Ehrenreich and Pfennig 2015; Levis and Pfennig 2016). Thus, the test of genetic assimilation requires documentation of progressive internalization of developmental controls of a trait whose expression was induced or maintained by interactions with the external environment in earlier evolutionary stages. Therefore, two elements are needed to investigate genetic assimilation (reviewed in West-Eberhard 2003): (1) assessment of the internalization of a trait's developmental controls and associated accommodation of a trait by other components of the phenotype and (2) assessment of the recurrence of the inductive environments.

\section{Coevolution of Carotenoids and Feather Structure as a Potential Case of Genetic Assimilation}

Three factors central to the evolution of carotenoid pigmentation of avian feathers make it particularly well suited to the study of genetic assimilation as an ongoing process. First, birds cannot produce carotenoids from noncarotenoids and thus must consume dietary (external) carotenoids to initiate their carotenoid metabolism during each molt (Buchecker 1982; Goodwin 1984; Brush 1990; Britton et al. 2004). Nevertheless, birds possess complex enzymatic machinery to convert external carotenoids into modified compounds that are incorporated into a growing feather (reviewed in Stradi 1998; McGraw 2006). The degree to which dietary carotenoids are modified before incorporation into the feather (measured as the number of enzymatic reactions) provides a mechanistic measure of internalization of carotenoid metabolism (fig. 1a). Some species deposit external carotenoids directly, without any internal biochemical conversion, whereas others subject consumed carotenoids to up to nine consecutive enzymatic modifications (often unique to a species) and express only highly derived, internalized carotenoids in their plumage (Higginson et al. 2016). Second, plumage carotenoids differ not only in length of biochemical pathways that link them to dietary inputs but also in biochemical dietary redundancy, the number of redundant enzymatic pathways by which derived carotenoids can be reached from their dietary precursors (fig. 1b). Over evolutionary time, this redundancy enables persistence and stability of highly derived carotenoids (end products of carotenoid metabolism) in species' plumage, despite fluctuations and diversity of dietary carotenoids (starting points; Badyaev et al. 2015). Thus, redundancy of pathways that produce a plumage carotenoid is the measure of a carotenoid's recurrence with other organismal traits. Third, carotenoids are accommodated by the complex developmental machinery of feather growth (reviewed in Lucas and Stettenheim 1972; Chuong 1993; Chen et al. 2015). The outcomes of such accommodation vary from short-term physical modifications of feather structure, to intricate reorganization of feather growth, to evolution of a specific feather microstructure to facilitate expression of particular carotenoids (Olson 1970; Troy and Brush 1983; Landeen and Badyaev 2012; García de Blas et al. 2013; LaFountain et al. 2015). Thus, the evolution of carotenoidbased ornamentation within avian lineages may be thought of as an arena for ongoing genetic assimilation, passing through stages of this process from phenotypic induction by external carotenoids to a progressively internalized metabolism of derived carotenoids and their subsequent integration with feather growth and restarting when the lineage switches to novel dietary precursors. Here, we group these evolutionary stages under the genetic assimilation hypothesis. 
(a)

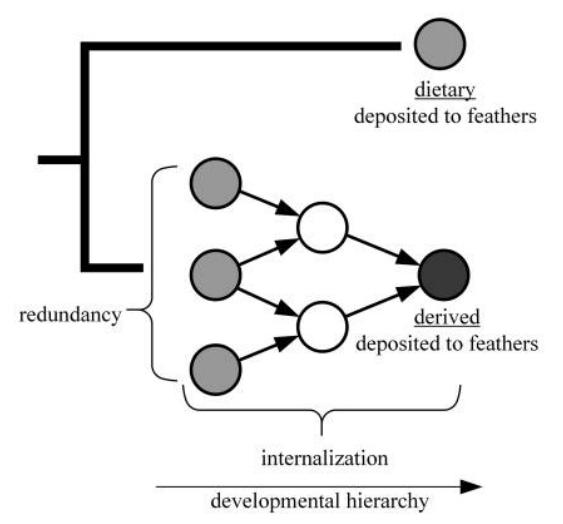

(b)

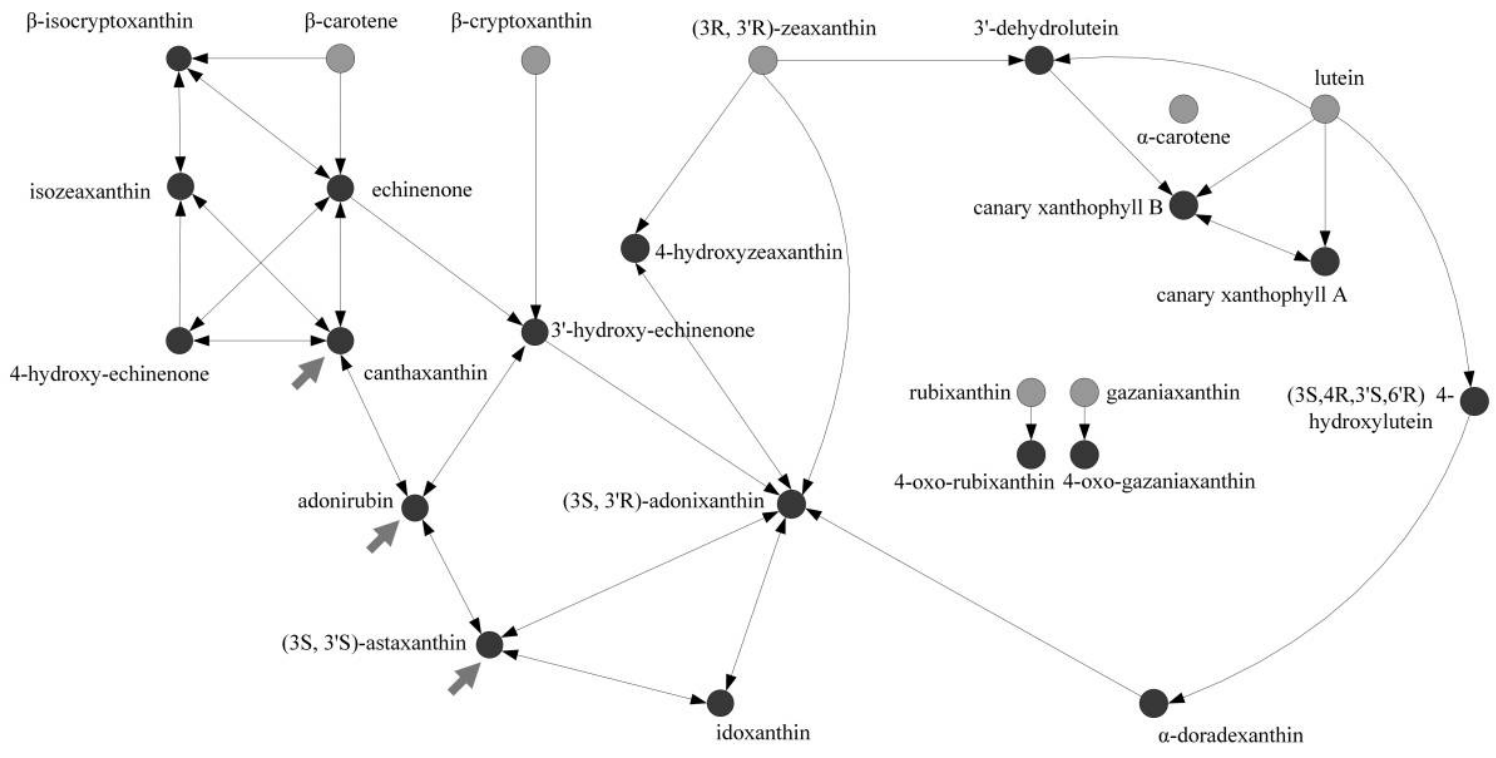

(c)

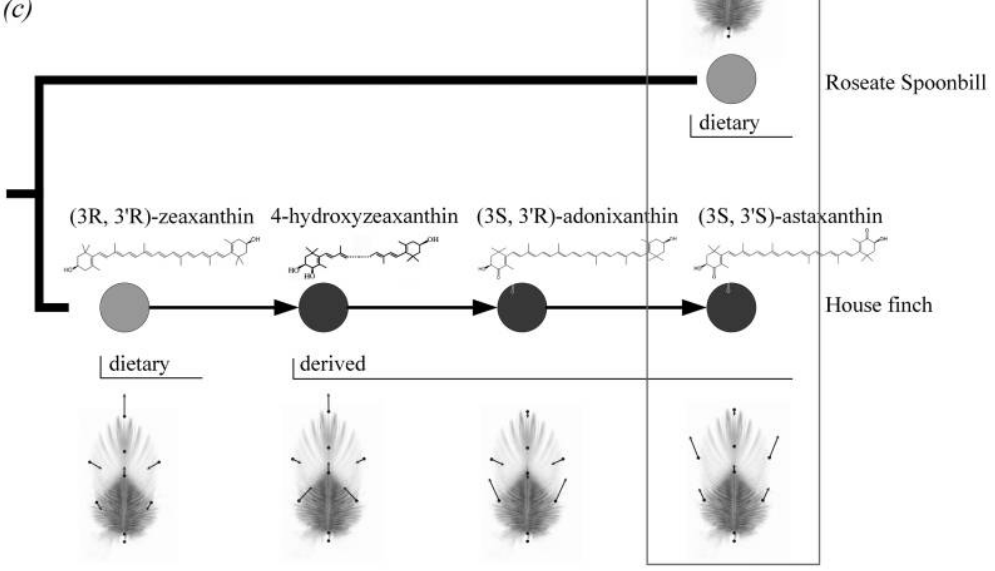




\section{Predictions of Genetic Assimilation Hypothesis for Coevolution of Carotenoids and Feather Structure}

Under the genetic assimilation hypothesis, an induced phenotype (i.e., feather structure physically modified by an external carotenoid) becomes internally stabilized (i.e., physical changes in feather structure occur without induction; fig. 2). A specific prediction of this process is that the effect of physical properties of a carotenoid on feather structure should depend on a carotenoid's incorporation into organismal homeostasis. Populations that are able to genetically assimilate the inclusion of carotenoids into feathers - such as by evolving their close integration with growth processes - will have higher fitness because they will benefit from greater and more efficient expression of a carotenoid without compromising other feather functions. Similarly, the effect of physical properties of the external carotenoids on feather structure should vary with the rarity of these compounds in a bird's diet: rarely encountered or novel carotenoids should induce more stochastic, stress-like modifications of feather structure, whereas a long-term association with the most recurrent carotenoids should be closely integrated with normal feather growth (fig. 2).

We test these predictions in ecological and evolutionary contexts in which carotenoids differ in the extent of their organismal internalization (i.e., biochemical conversion) and recurrence (i.e., frequency of consumption and biochemical redundancy). First, we test whether modification of feather structure by carotenoids of different molecular weight (a proxy for size) depends on a carotenoid's internalization and ecological and biochemical recurrence. Further, we capitalize on the fact that carotenoids that are dietary in one species can be highly derived (i.e., internalized) in another species and vice versa (fig. 1c) and compare feather modification by the same carotenoids in distinct (a)

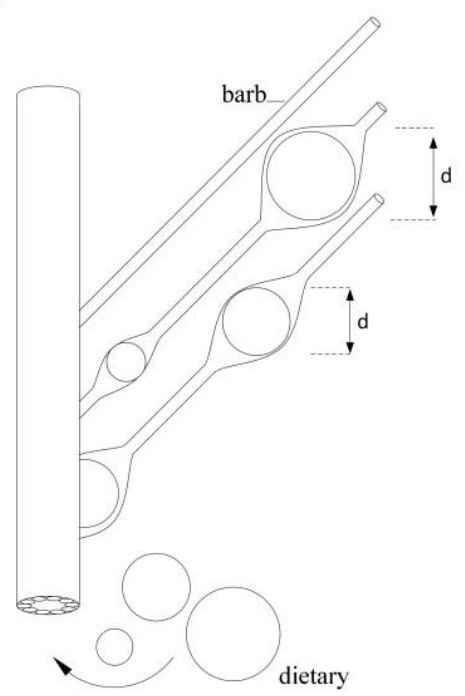

(b)

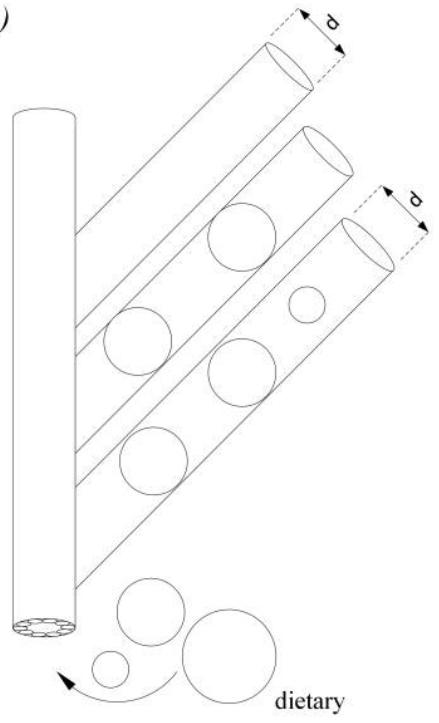

(c)

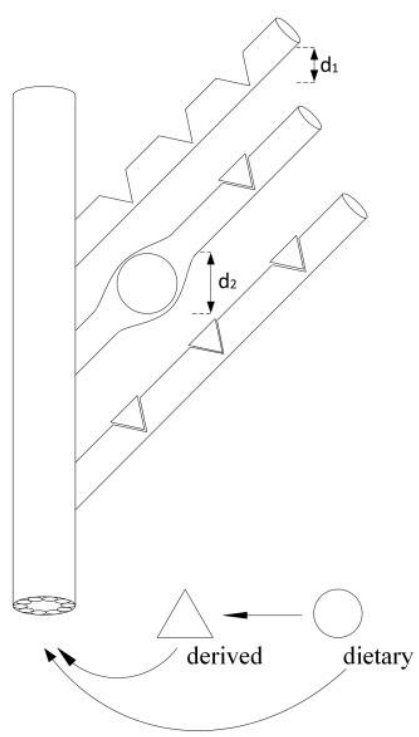

Figure 2: Evolutionary stages of genetic assimilation of carotenoids into a growing feather. $a$, Physical modification of feather structure by accommodation of dietary carotenoids. Displacements (indicated by change $d$ in barb diameter) is proportional to physical properties (e.g., size) of absorbed carotenoids. $b$, Genetic accommodation of induced change $d$ in feather structure that enables reliable expression of carotenoids. Under this scenario, change $d$ is produced without direct induction (upper modified barb without carotenoids). Genetic accommodation can be produced either by lowering the sensitivity of carotenoid uptake (enabling intake of carotenoids of diverse sizes; lower barb) or by genetic stabilization of induced patterns (a), resulting in selective uptake and accommodation of only specific carotenoid types (middle barb). $c$, Genetic assimilation of metabolically derived carotenoids. Feather constitutively expresses specific structural modifications needed for accommodation of particular internally metabolized carotenoids, regardless of the presence of these carotenoids $\left(d_{1}\right.$; filled and open triangular niches). Accommodation of dietary carotenoids results in distinct feather modifications $\left(d_{2}\right.$; middle barb with circle). When a species expresses a mixture of internally metabolized and dietary carotenoids, this scenario enables assessment of the relative importance of recurrence (which is the same for upstream and downstream carotenoids in a metabolic sequence; fig. 1b) and organismal internalization (degree of metabolic elaboration) for the evolution of $d$. Comparisons of the patterns and magnitude of displacement $(d)$ within and across these stages provide inference into the likelihood of genetic assimilation as a process behind the evolution of specialized carotenoid-bearing feathers. Results of this study are most consistent with scenario in $c$. 
evolutionary contexts. We predict that the same carotenoid types will be more integrated with feather growth when in a derived state versus in a dietary state. We then take advantage of naturally occurring variation in morphology and carotenoid composition of feathers across ornamental parts, individuals, and populations within a species and compare the effect of dietary and derived carotenoids on feather structural modification. The genetic assimilation hypothesis predicts that patterns of variation in feather structure across individuals and populations should be most concordant with those caused by the effects of derived carotenoids. Finally, we assess feather accommodation of dietary and derived carotenoids by comparing their integration with feather growth. The genetic assimilation hypothesis predicts that the most recurrent and derived carotenoids should have a closer integration with elements of feather growth compared with unique derived or dietary compounds. Using one of the largest data sets ever assembled for such tests, we show that the effect of carotenoid type on feather structure depends on internalization of the carotenoid metabolism and its biochemical and environmental recurrence, the pattern predicted by the genetic assimilation hypothesis.

\section{Material and Methods \\ Data Collection}

We quantified the concentration of 16 carotenoids (appendix, available online) in 14,637 feathers of 1,018 male house finches (Haemorhous mexicanus) from 11 study populations in Montana and Arizona (table A1; tables A1-A4 are available online) and in 77 feathers of 19 male roseate spoonbills (Platalea ajaja; table A2). The house finch samples were collected in 2002-2014 during an ongoing study of free-living, individually marked birds (general protocols in Badyaev and Vleck 2007). The spoonbill samples were from two collections (American Museum of Natural History and University of Arizona Natural History Museum) as well as from the breeding colony in Reid Park Zoo (Tucson, AZ). In finches, 15 ornamental feathers (five from each of the three ornamental areas [crown, breast, and rump]; fig. 1 in Badyaev and Landeen 2007) were taken from each bird on capture. In spoonbills, five feathers were taken from two ornamented areas (breast and shoulder).

In house finches, dietary carotenoids are lutein, (3R, $\left.3^{\prime} \mathrm{R}\right)$ zeaxanthin, $\beta$-cryptoxanthin, $\alpha$-carotene, gazaniaxanthin, rubixanthin, and $\beta$-carotene, whereas adonirubin, $\left(3 \mathrm{~S}, 3^{\prime} \mathrm{S}\right)$ astaxanthin, $\left(3 \mathrm{~S}, 3^{\prime} \mathrm{R}\right)$-adonixanthin, $\beta$-isocryptoxanthin, 3'-dehydrolutein, echinenone, 3'-hydroxy-echinenone, 4oxo-rubixanthin, and canthaxanthin are derived from dietary carotenoids by 1-4 enzymatic conversions (fig. 1b). Three of the most derived carotenoids in house finches$\left(3 \mathrm{~S}, 3^{\prime} \mathrm{S}\right)$ astaxanthin, adonirubin, and canthaxanthin - are dietary in roseate spoonbills (fig. $1 b$; appendix S2 in Badyaev et al. 2015). We obtained molecular weights and other physical properties of carotenoids from Britton et al. (2004; table A3). Carotenoid extraction, identification, and quantification are described by Higginson et al (2016). Concentrations of all carotenoids were logarithmically transformed and the normal distribution confirmed with Proc Univariate in SAS 9.4.

\section{Feather Morphometrics and Growth}

Feathers were digitized with a modified Epson Perfection 1660 PhotoScanner (Long Beach, CA) at 1,000 dots per inch (sample sizes in table A1). In each house finch feather, at full magnification, we digitized nine homologous landmarks. These landmarks were selected because previous empirical and modeling studies found that their displacement reflects changes in feather shape and structure, including those caused by unfurling and transformation during transition from tubular to two-dimensional structure in feather ontogeny and those associated with accommodation of carotenoids during growth (references in Badyaev and Landeen 2007). The landmarks were (fig. A1, available online) (1) base of feather calamus, (2) base of feather rachis, (3) structural boundary along rachis, (4) end of rachis, (5) tip of feather, $(6,7)$ the longest rachis (also the widest part of feather), and $(8,9)$ angle formed by rachis. Because the entire feather is pigmented in roseate spoonbills, landmarks 8 and 9 were omitted for this species. Two observers obtained landmark coordinates for the house finch data set, and a single observer obtained landmark coordinates for the spoonbill data set with tps software (F. J. Rohlf, State University of New York, Stony Brook). Rachis curvatures were standardized using landmarks 1 and 4 for all feathers with tpsUtil software (F. J. Rohlf, State University of New York, Stony Brook) and SAS/IML9.4 script. To obtain landmark displacements due to variation in individual growth parameters, we used simulated feather growth and unfurling beginning with the emergence of the initial barb ridges in the follicle (Badyaev and Landeen 2007).

\section{Data Analyses}

We used Procrustes superimposition (Bookstein 1996; Dryden and Mardia 1998) to characterize feather shape variation. Separate Procrustes superimpositions were done for each species, because two landmarks used for house finch feathers were not used for spoonbill feathers. Feather centroid sizesthe square root of the sum of squared distances of each landmark from the centroid-are in table A1.

Variance in the set of optimally aligned landmark configurations (hereafter Procrustes coordinates) was then par- 
titioned using ANOVA models. Individual identity was a random effect, and ornamental area (crown, breast, rump) was nested within an individual term. Degrees of freedom for the Procrustes ANOVA were calculated following Goodall (1991), and we used permutation methods described by Klingenberg and McIntyre (1998) to test ANOVA effects. To partition the effects of each landmark on overall variation in feather shape, we summed mean squares of their $x$ and $y$ coordinates and computed variance components of mean squares according to the expected mean squares for each of the effects (fig. A1; Badyaev and Foresman 2000). We analyzed the covariance matrices of the Procrustes coordinates and-on the basis of the expected mean squares-computed matrices of sums of squares and cross products (SSCP) for each effect and carotenoid type.

The measurement error due to variation between the two observers (for the finch data set) was assessed for the subsample of 10 individuals (breast feathers only) that had been measured twice and analyzed through a Procrustes two-factor ANOVA. The effect of interobserver measurement error was $<3 \%$ of the individual identity effect (mean squares 0.68 vs. 33.7 ) and was therefore negligible.

Patterns of variation within feathers were examined and displayed using principal component (PC) analysis for each of these covariance matrices, which shows the joint pattern of landmark displacement along the direction of maximum variance in shape and essentially summarizes nine-dimensional shape space for each feather to a single dimension. PC1s accounted for $>60 \%$ of variation for all carotenoid compounds, so to visualize landmark covariation, we graphically represented PC1s as displacement from landmark consensus position (obtained for each species separately with tpsUtil) with a vector connecting the consensus and final coordinates. To examine similarity in patterns of landmark displacement, we computed the angles between the PC as the arc cosine of the inner product of the two eigenvector elements. We used a bootstrap test (Efron and Tibshirani 1993) with $n=10,000$ iterations to test whether pairs of PC1s were identical (i.e., angle is 0 ). To do this, we generated a null distribution of angles and then performed resampling with replacement among PC scores of individual specimens.

\section{Parameters Used in This Study}

The genetic assimilation hypothesis predicts that the effect of physical properties of carotenoids on feather structure should depend on carotenoids' metabolic modification, their environmental recurrence, and biochemical redundancy. We assessed changes in feather structure as displacement of Procrustes coordinates of landmarks that are known to closely reflect ontogenetic and evolutionary transformations of feathers. To simultaneously evaluate the magnitude and coordination of landmark displacement, we used two parameters: total displacement of landmarks (assessed as the trace of SSCP matrix) and feather reorganization (measured as the first eigenvalue $\lambda_{1}$ ). The total displacement was the total absolute magnitude of displacement due to a particular effect, without regard to coordination or similarity of the displacement across landmarks. The feather reorganization specifically reflected directions of maximal covariance among the landmarks: $\lambda_{1}$ thus represents coordinated changes in feather shape due to each carotenoid type. These metrics gave qualitatively identical results to the assessment of sum of squared covariances among landmarks and the partial least squares method, correspondingly (Klingenberg et al. 2001). The genetic assimilation hypothesis predicts that metabolically derived, frequently consumed, or biochemically redundant carotenoids will cause more coordinated displacement of landmarks and a lesser magnitude of landmark displacement than dietary, novel, or biochemically unique carotenoids of the same molecular weight. These patterns are expected from greater integration of derived carotenoids into processes of feather growth and differentiation, an assumption that should be reflected in greater concordance of the displacement they cause with those accompanying feather transformation during growth. Further, the greater total displacement measure, by definition, reflects dissimilar displacements of landmarks among individuals and is thus a measure of stochastic variation in the landmarks in relation to carotenoid type deposition, when all other covariates (populations, individuals, and ornamental part) are statistically controlled.

We calculated total displacement and feather reorganization separately for each carotenoid type and for the groups of dietary and derived carotenoids. For the latter, the total concentration of all compounds in a group was analyzed as a single effect; for the former, the relative effects of concentrations of individual carotenoid types were calculated. Thus, the dietary and derived group effects were not sums of the effects of their individual components. Occurrence of a carotenoid was a ratio of sampled birds that had the carotenoid type (table A3). To measure biochemical dietary redundancy of a compound, we determined the total number of unique noncyclical pathways among each of the expressed compounds in the species network (44 reactions between 24 compounds; fig. 1b) and each of the dietary carotenoids in this network (lutein, zeaxanthin, $\beta$-carotene, $\beta$-cryptoxanthin, $\alpha$-carotene, rubixanthin, and gazianaxanthin). We calculated redundancy with a breadth-first search algorithm (Moore 1959) using a modified code (http://stackoverflow.com/questions /58306/graph-algorithm-to-find-all-connections-between-two 
-arbitrary-vertices). Dietary redundancy was the sum of all of the unique noncyclical pathways between a derived carotenoid and each of the dietary inputs (table A4).

\section{Results}

\section{Feather Modification in Relation to Carotenoid Size, Dietary Redundancy, and Occurrence}

Although house finch populations were similar in ornamental feather sizes and total concentration of carotenoids, distribution of dietary and derived carotenoids varied widely among the three ornamental body parts within and across populations (table A1). For example, finches in Montana populations had a different distribution of rubixanthin among ornamental parts compared with finches in the Arizona populations (table A1). Finches in the Montana populations had up to four times higher concentrations of astaxanthin and two times lower concentrations of $\beta$-isocryptoxanthin than did finches in Arizona populations (table A1). Accommodation of dietary carotenoids caused greater landmark displacement but lesser feather reorganization than did accommodation of derived carotenoids (fig. $3 a, 3 b$ ). Total landmark displacement increased with molecular weight of dietary carotenoids (fig. $3 a ; F=$ $\left.35.62, b_{\mathrm{ST}}=0.93, t=5.97, P=.002\right)$ but did not vary with molecular weight of derived carotenoids (fig. $3 a ; F=$ $\left.0.01, b_{\mathrm{ST}}=0.03, t=0.1, P=.92\right)$. In contrast, feather reorganization did not vary with molecular weight of incorporated dietary carotenoids (fig. $3 b ; F=0.21, b_{\mathrm{ST}}=$ $0.19, t=0.45, P=.60)$ but did increase with molecular weight of derived carotenoids (fig. $3 b ; F=17.57, b_{\mathrm{ST}}=$ $0.84, t=4.19, P=.004)$. Controlling for the effect of molecular weight, more commonly used dietary carotenoids had a lesser effect on landmark displacement than did rarely used dietary compounds (fig. $3 c$; Kendall's $\tau=$ $-0.81, P=.01)$. In derived carotenoids, the frequency of occurrence did not correlate with total landmark displacement (Kendall's $\tau=0.33, P=.21$ ). Controlling for the effect of molecular weight, biochemically redundant carotenoids had a weaker effect on the magnitude of landmark displacement (fig. $3 d$; $b_{\mathrm{ST}}=-0.93, t=-6.76, P=$ .003 ) but a stronger effect on feather reorganization than did biochemically unique carotenoids (fig. $3 d ; b_{\mathrm{ST}}=0.86$, $t=4.47, P=.003)$.

\section{Carotenoid-Induced Feather Modifications across Ornaments, Individuals, Populations, and Species}

Patterns and magnitude of feather shape modification associated with accommodation of carotenoids in both species are shown in figures 4 and A1. We compared patterns of variation in feather shape among ornamental body parts, individuals, and populations of the house finch to the variation in feather shape caused by accommodation of dietary and derived carotenoids (fig. $5 a-5 c$ ). Feather shape variation across ornamental parts was not distinguishable from variation caused by accommodation of dietary carotenoids as a group, although contribution of individual carotenoids differed (fig. 5a). Feather shape variation among individuals was closely associated with changes caused by derived carotenoids and to a lesser extent by dietary carotenoids (fig. 5b), and shape variation among populations was highly concordant with displacement caused by derived carotenoids (fig. 5c). Thus, feather differences among populations and individuals were largely attributable to their accommodation of derived carotenoids, whereas feather differences within an individual were largely due to their differential accommodation of dietary carotenoids. Because individual carotenoids in both dietary and derived groups varied in their contribution to these effects (fig. $5 a-5 c$ ), we examined whether such within-group variation was associated with molecular weight of individual carotenoids, their occurrence, or dietary redundancy. We selected the best predictor of within-group variation for each of these three factors (stepwise regression; fig. $5 d-5 f$ ). The frequency of consumption was the best predictor of the dietary carotenoid contribution to feather shape variation among ornamental parts: more frequently consumed carotenoids contributed more to the variation than did rarely consumed carotenoids (fig. $5 d$; Kendall's $\tau=0.62, P=.05$ ). Dietary redundancy was the best predictor of the derived carotenoid contribution to both differences among individuals and populations; in both cases, biochemically unique carotenoids accounted for most variation in feather shape among individuals (fig. $5 e$; Kendall's $\tau=-0.50, P=.05$ ) and populations (fig. $5 f$; Kendall's $\tau=-0.56, P=.03$ ). The effects on feather shape of the three compounds that were derived in the house finch, but dietary in the spoonbill, were more similar when these compounds were dietary (in the spoonbill) and most distinct when they were derived (in the house finch; fig. $6 c$ ).

\section{Concordance of Carotenoid-Induced Modification and Feather Growth}

Feather shape changes caused by accommodation of derived carotenoids were statistically indistinguishable (i.e., upper dashed line in fig. 7) from feather shape transformation due to changes in absolute growth rate, feather barb addition rate, barb number, and feather expansion angle, whereas feather shape changes caused by accommodation of dietary carotenoids were most similar to transforma- 
(a)

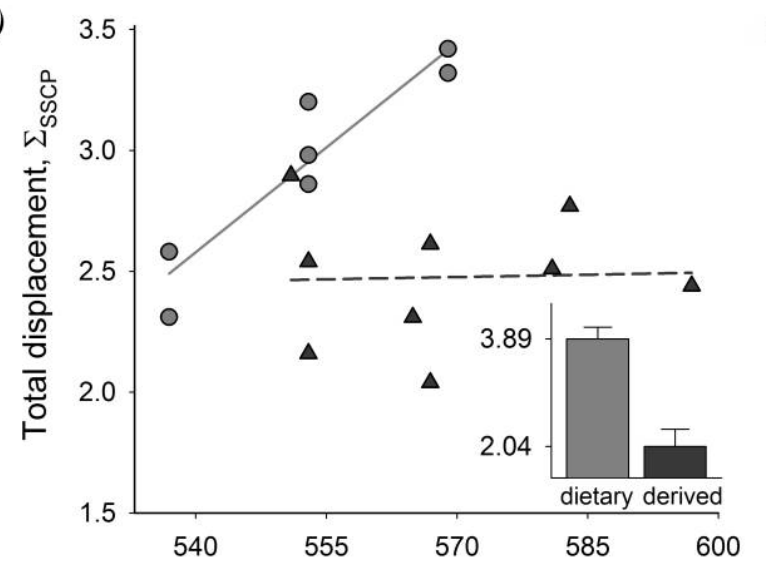

(b)

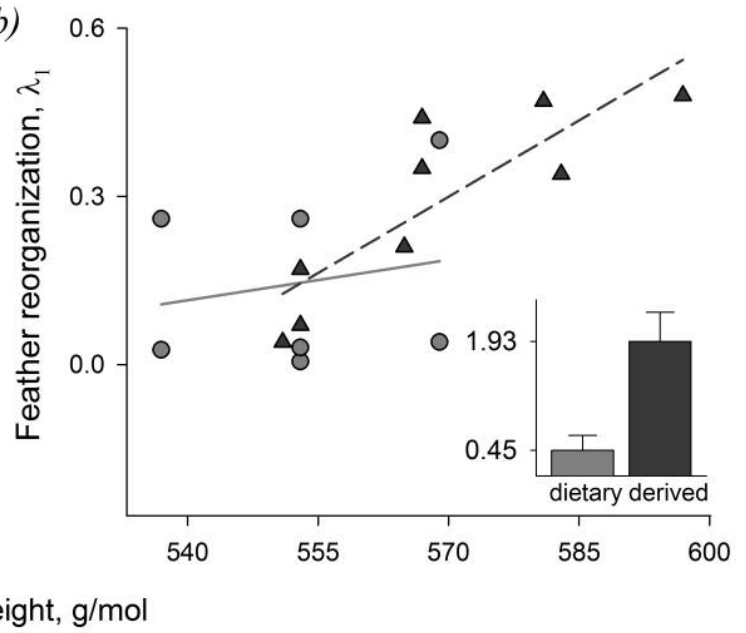

(c)

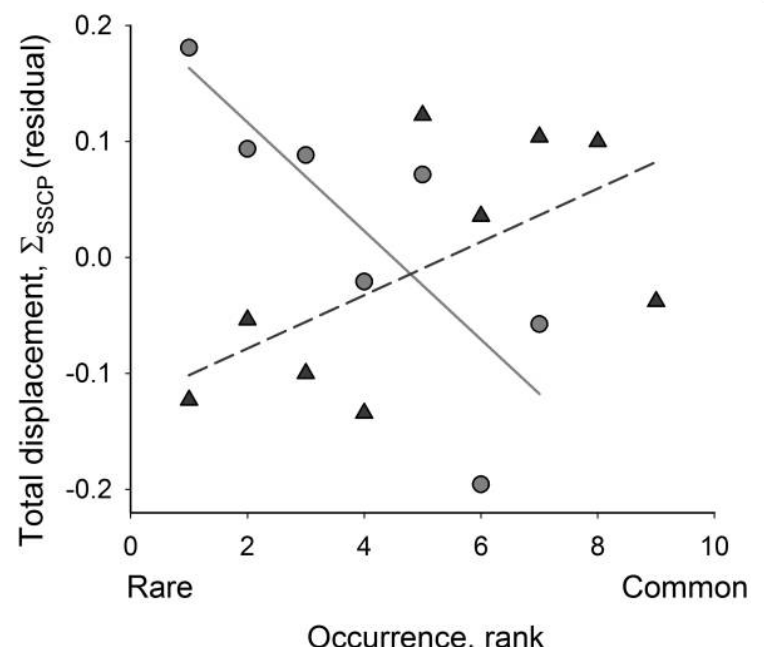

(d)

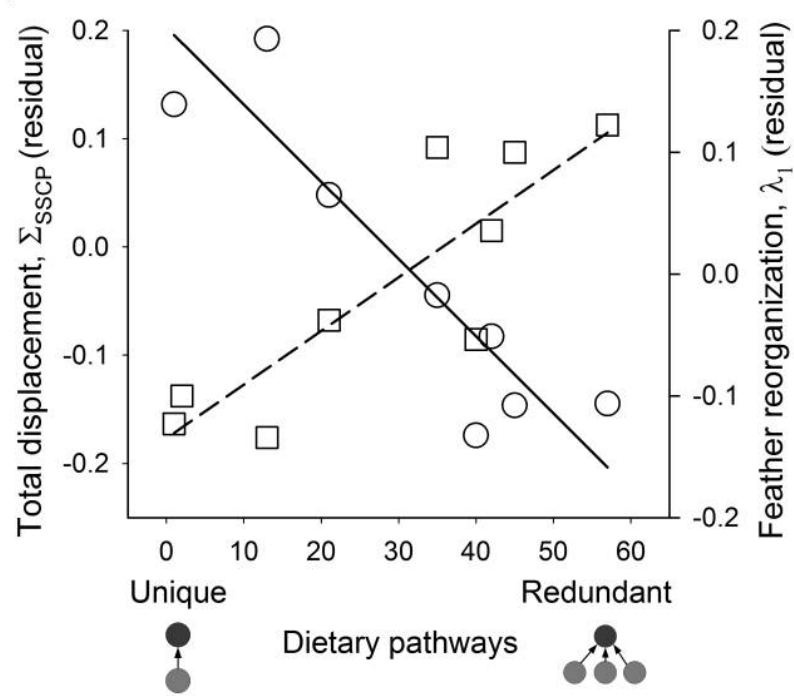

Figure 3: Feather modification in relation to carotenoid's molecular weight depends on carotenoid cooption, frequency of occurrence, and dietary redundancy. $a$, Total landmark displacement increased with carotenoid size for dietary (circles) but not for derived (triangles) carotenoids. Inset, dietary carotenoids had a greater effect on total landmark displacement than derived carotenoids (mean \pm SE). SE was derived by bootstrap with resampling of individual observations within a group. $b$, Derived carotenoids had a greater effect on feather reorganization (inset) than did dietary carotenoids, and this effect increases with carotenoid size. $c$, Controlling for the effect of molecular weight, more commonly consumed dietary carotenoids had a lesser effect on landmarks' total displacement than did rarely consumed dietary carotenoids (solid line, circles). In derived carotenoids, total landmark displacement tended to increase with the frequency of occurrence (dashed line, triangles). $d$, Controlling for the effect of molecular weight, biochemically redundant carotenoids had a greater effect on feather reorganization (dashed line, squares) but a lesser effect on total landmark displacement than did biochemically unique carotenoids (solid line, circles). Biochemical redundancy was calculated for derived compounds only (table A3, available online). SSCP, sums of squares and cross products.

tions caused by the increase in barb diameter and feather expansion angle (fig. 7). Overall, derived carotenoids were more closely integrated with feather growth parameters than dietary carotenoids and were particularly closely integrated with growth rate (fig. 7).

\section{Discussion}

Developmental processes are evolving composites of both physical and genetic mechanisms (Oster and Alberch 1982; Müller 2007; Newman 2012). The relative importance 

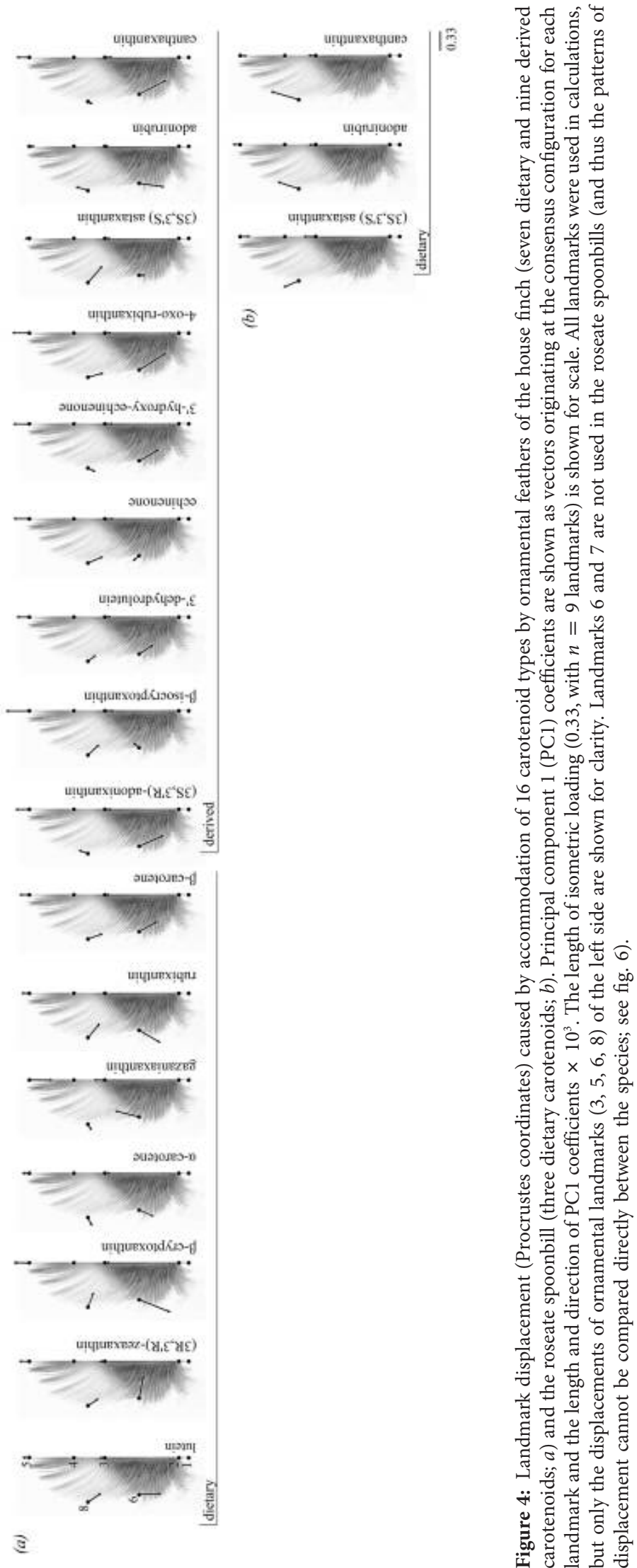

This content downloaded from 150.135.119.147 on August 22, 2017 16:54:18 PM All use subject to University of Chicago Press Terms and Conditions (http://www.journals.uchicago.edu/t-and-c). 
(a)

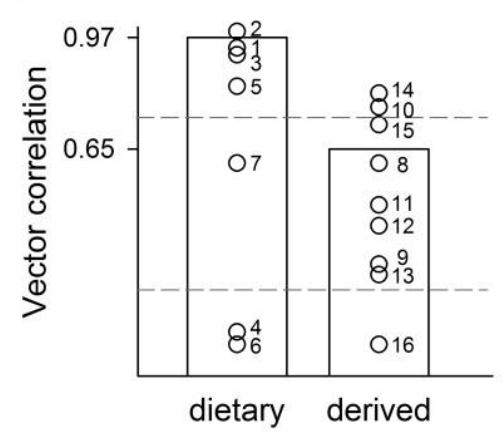

(d)

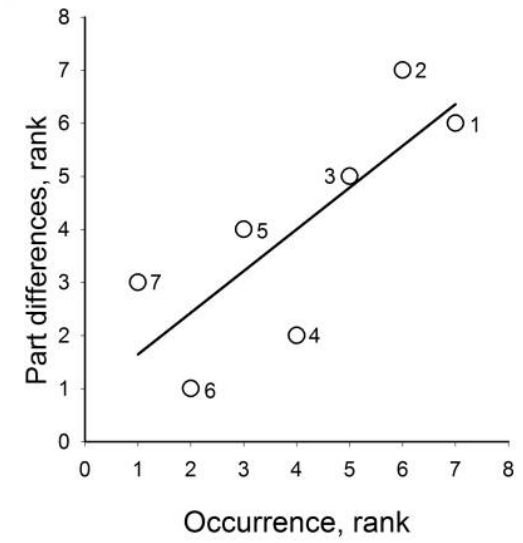

(b)

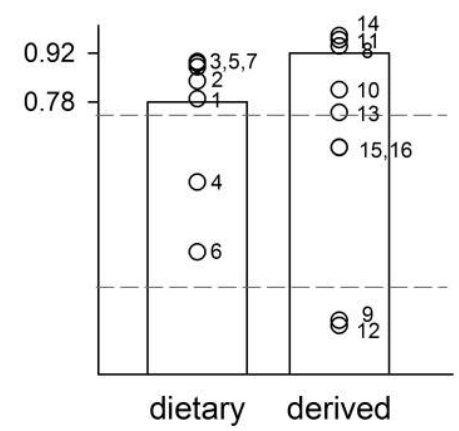

(e)

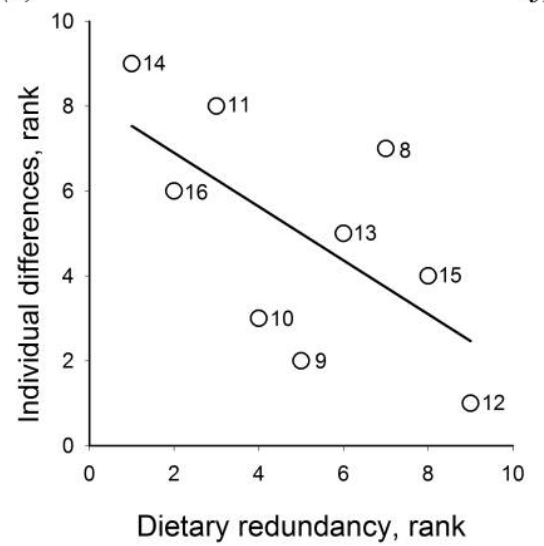

(c)

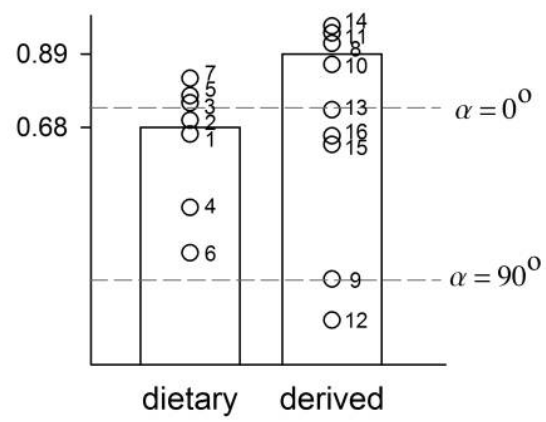

(f)

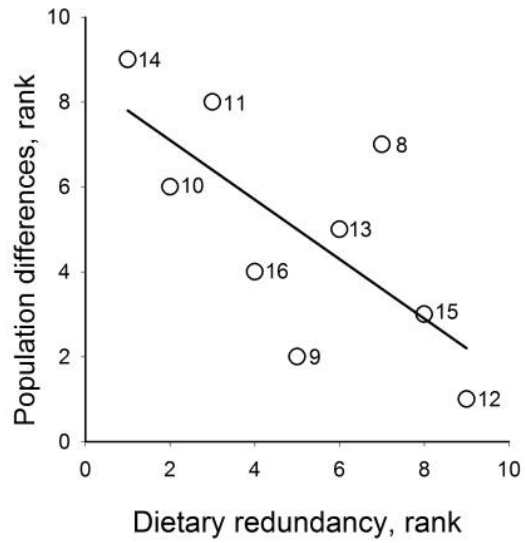

Figure 5: $a-c$, Concordance of feather modifications caused by accommodation of dietary and derived carotenoids and feather variation among ornamental parts $(a)$, individuals $(b)$, and populations $(c)$ in the house finch. Shown are vector correlations between principal component 1 (PC1) of Procrustes mean squares of each effect. Bars are means for the groups of dietary and derived carotenoids. Circles show partial displacement due to individual carotenoid types (legend for numbers in table A3, available online). Vector correlations above upper dashed line indicate PC1 angles that are not different from $0^{\circ}$ (i.e., completely concordant with each other), correlations below lower dashed line indicate angles that are not different from $90^{\circ}$ (i.e., completely discordant from each other), and vector correlations between dashed lines show angles different from both $0^{\circ}$ and $90^{\circ} . d-f$, Correlates of variation in individual carotenoid types that contribute most strongly to differences between ornamental parts $(d)$, individuals $(e)$, and populations $(f)$.

of these mechanisms varies with the evolutionary stage and recurrence of the environmental setting in which a developing structure functions. When phenotypic variation produced by physical factors becomes beneficial (e.g., acquires the greatest fit with prevalent environment) in the most recurrent organism-environment association, its production can be stabilized by accumulation of more reliable genetic machinery and molecular hierarchies (Chetverikov 1926; Oyama 1988; Stebbins and Hartl 1988; Newman and Müller 2000). Conversely, a lack of fit between a structure and its current environment exposes phenotypic variation previously masked by genetic regulation (Gibson and Dworkin 2004; Hermisson and Wagner 2004; Badyaev 2005).

A particular challenge has been to study these phenomena as a continuous process, and here we argue that the coevolution of carotenoid compounds and the feather struc- ture that they induce offers such an opportunity for several reasons. First, some carotenoids are deposited into a feather follicle directly and passively, while others form carotenoproteins or esterified complexes with feather proteins, often with highly specific directional binding (Fox 1962; Britton et al. 1982; Tyczkowski and Hamilton 1986; García de Blas et al. 2013). Correspondingly, modification of a feather's keratin matrix caused by carotenoid accommodation varies from stochastic physical displacements caused by novel or incidentally digested carotenoids to highly specialized and selective integration of a particular carotenoid type into the feather structure (Gaier et al. 1991; Stradi 1998; Wyss et al. 2001; García de Blas et al. 2011; Mendes-Pinto et al. 2012). Second, feathers perform a variety of functions in addition to carotenoid display; thus, selection should favor the most efficient and beneficial accommodation of caroten- 
(a)

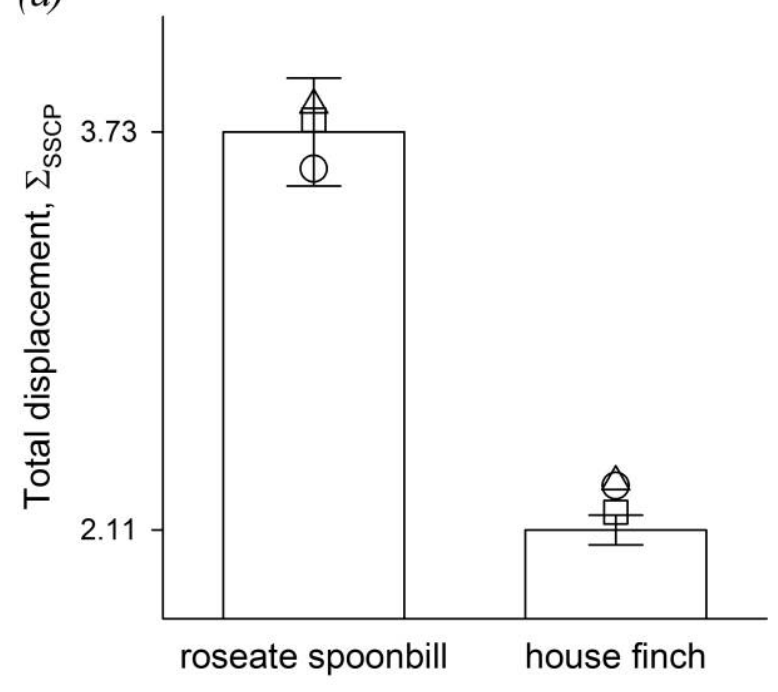

(b)

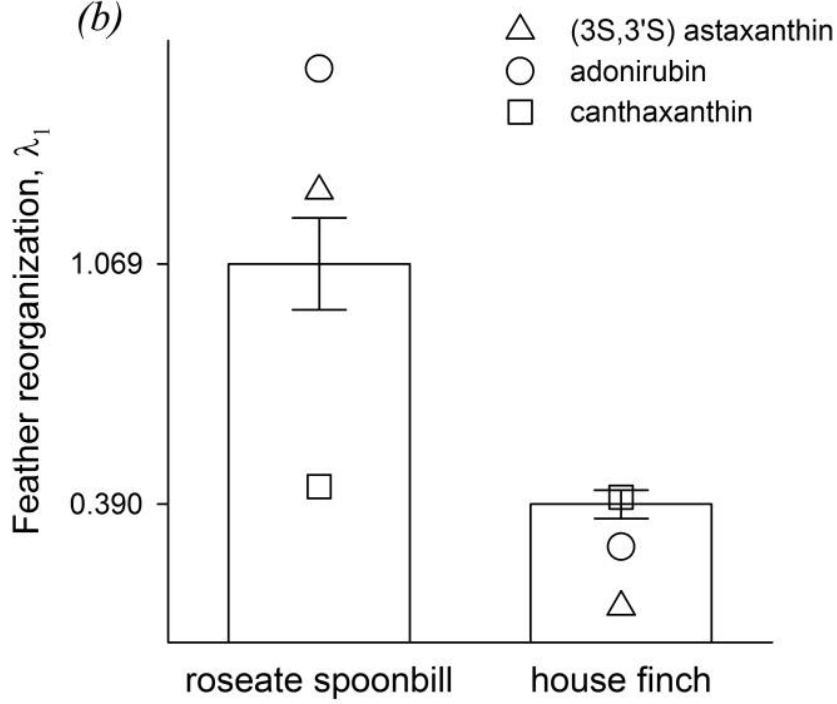

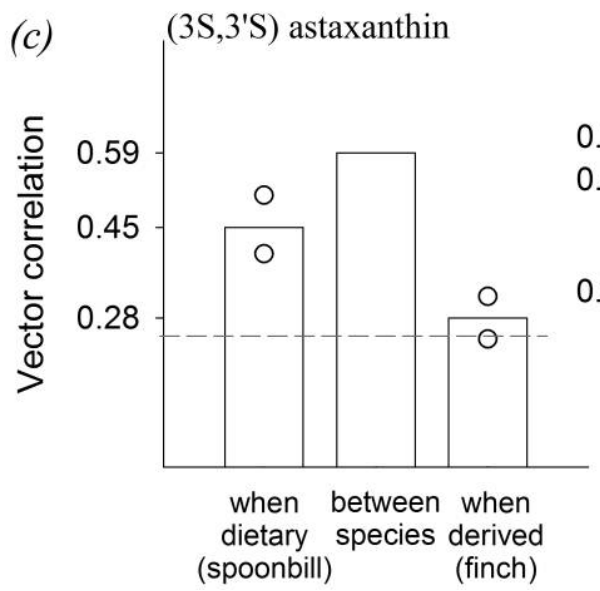
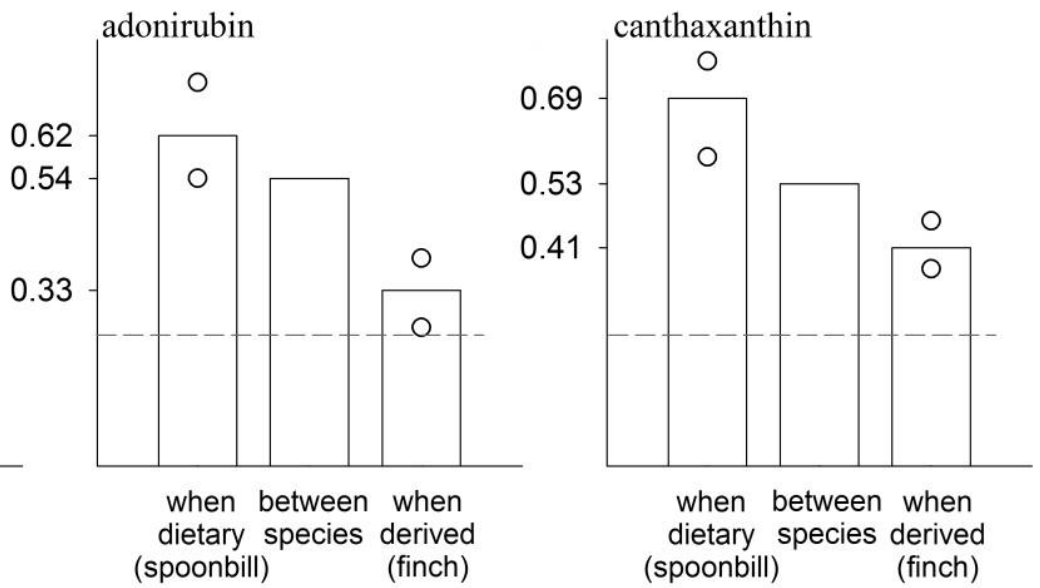

Figure 6: Effect (mean $\pm \mathrm{SE}$ ) of carotenoids shared between the study species (derived in the house finch and dietary in the roseate spoonbill) on magnitude of landmark displacement $(a)$ and feather reorganization $(b)$. $c$, Concordance of feather modifications caused by accommodation of three carotenoid compounds when these are dietary (left bar; in spoonbills), derived (right bar; in finches), and between dietary and derived states of the same carotenoid (center bar; i.e., between species). Effects of carotenoid type on feather shape are most similar when carotenoids are dietary and most dissimilar when they are derived. Shown are vector correlations (also labels on $Y$-axis) between principal component 1 ( $\mathrm{PC} 1$ ) of displacements due to each carotenoid type (left and right bars) and between PC1 of the same carotenoids between species. Correlations above dashed line indicate angles different from $90^{\circ}$ (i.e., correlations different from 0). SSCP, sums of squares and cross products.

oids that does not compromise the other functions of the feather. Finally, feather keratinogenesis, carotenoid transport, and follicle absorption might share hormonal regulation (Dawson and Sharp 1998; Gossage et al. 2000; Badyaev and Vleck 2007) that could facilitate their integration.

Our results are consistent with predictions of the genetic assimilation hypothesis (fig. 2). Feather accommodation of carotenoids depended on a carotenoid's organismal cooption; derived carotenoids caused less stochastic variation in feather shapes (fig. $3 a$, inset) and were integrated closer with feather growth than were dietary carotenoids of similar molecular weight (fig. $3 b$, inset; fig. 7). These patterns were driven by recurrence of these compounds: more widespread dietary carotenoids and more biochemically redundant derived carotenoids caused less stochastic variation in feather shape than did rarely used or biochemically unique compounds (fig. $3 c, 3 d$ ). Further, the effect of carotenoids shared between the study species on feather shape was most similar when these compounds were dietary than when these compounds were derived (fig. 6c). 


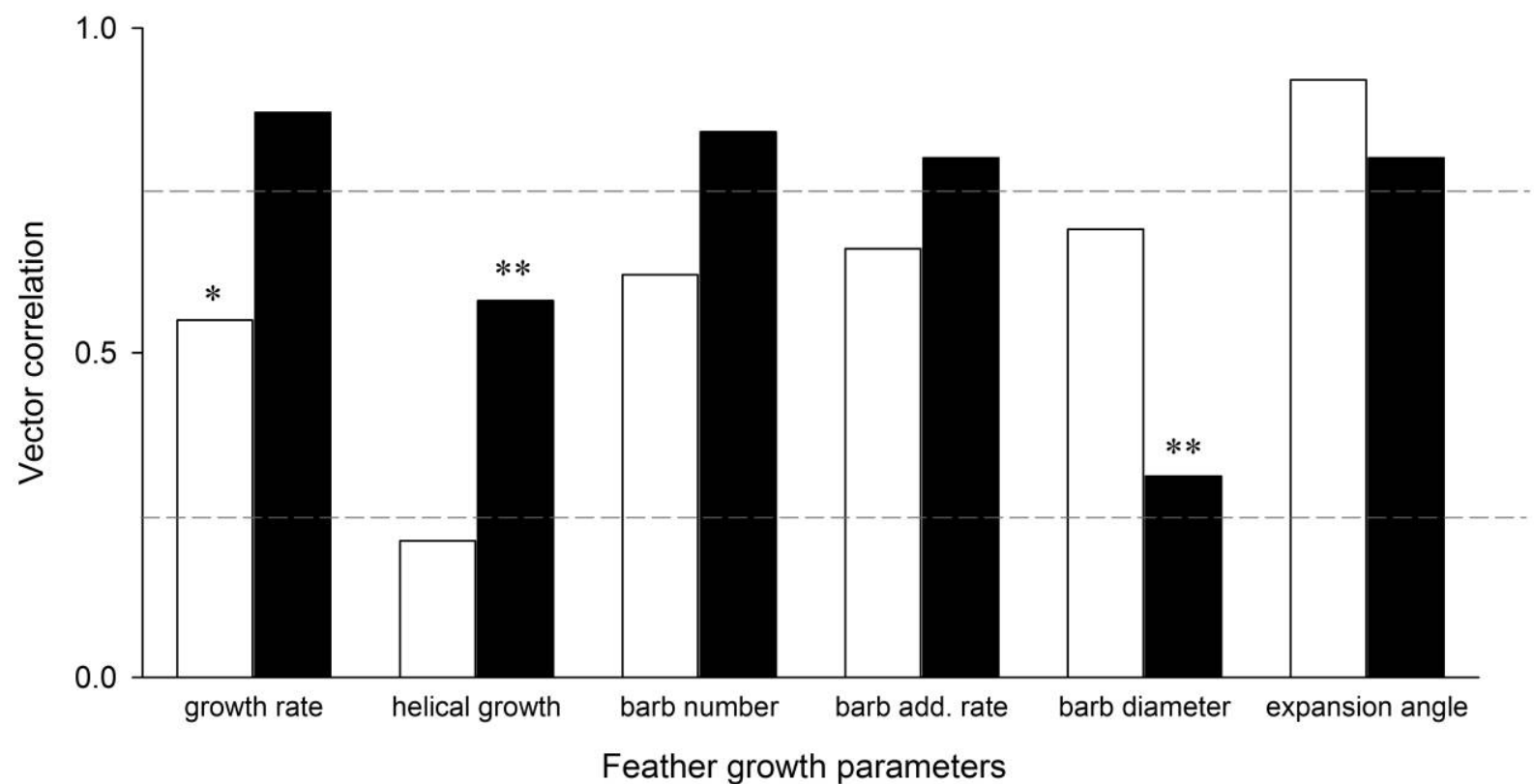

Figure 7: Concordance of feather modifications caused by accommodation of dietary (open bars) and derived (filled bars) carotenoids and variation produced by feather growth in the house finch. Shown are vector correlations between principal component 1 (PC1) of displacement caused by carotenoids (from fig. 4) and PC1 of displacement due to growth parameters (from Badyaev and Landeen 2007). Asterisks show differences between dietary and derived carotenoids for each growth parameter (from left to right: absolute growth rate, helical growth, initial barb number, barb addition rate, barb diameter, rachis expansion angle). Vector correlations above upper dashed line indicate PC1 angles that are not different from $0^{\circ}$ (i.e., completely concordant with each other), correlations below lower dashed line indicate angles that are not different from $90^{\circ}$ (i.e., completely discordant from each other), and vector correlations between dashed lines show angles different from both $0^{\circ}$ and $90^{\circ}$. One asterisk, $P<.1$; two asterisks, $P<.05$.

Genetic assimilation of these carotenoids results in progressively closer integration with components of feather growth (fig. 7) and thus greater evolutionary divergence in feather shape between the species when these carotenoids are in the derived state.

That structural modification of carotenoid-bearing feathers evolves through genetic assimilation has been proposed before, on the basis of observations of parallelism in the effect of dietary and derived carotenoids on feather structure (Hudon 1991; Price et al. 2003), the finding that carotenoid-specific modification of feather structure may not require direct induction (Landeen and Badyaev 2012), and the observation that carotenoid uptake and feather growth correlate, likely because of shared regulation by pituitary prolactin (e.g., Badyaev and Duckworth 2005). However, stronger inference into genetic assimilation requires an independent assessment of internalization of controls behind phenotypic change and an assessment of the recurrence of the inducing effect. The perspective presented here satisfies both of these requirements (fig. $1 b, 1 c$ ) and also uncovers a plethora of evolutionary and ecological contexts in which to observe genetic assimilation as a process. This is because metabolic modification and persistence of carotenoid compounds vary widely across species: highly metabol- ically derived internal carotenoids in one species are often external dietary compounds in another (e.g., Badyaev et al. 2015), allowing for direct comparison of a carotenoid effect on feather structure while controlling for its physical properties. Moreover, birds show repeated and frequent convergence in the use of specific carotenoids in their plumage across phylogenetically distant taxa, often from different starting points and in different feather morphologies (e.g., Prager and Andersson 2010; Friedman et al. 2013). Versatility and redundancy of feather growth controls - where each feather follicle harbors an ability to produce an exceptional array of feather shapes and structures and where similar modifications in feather shape can be produced by distinct combinations of growth controls (Price et al. 1991; Prum and Williamson 2001; Lin et al. 2013; Chen et al. 2015) - can enable both efficient accommodation of carotenoids and evolutionary retention and diversification of the changes in feather structure they induce. Further, the genetic assimilation process preserves a wide range of genetic variants even in the absence of selection for these variants (Schlichting and Pigliucci 1998) and thus enables variable environmental inputs to be channeled into consistent developmental outcomes, a scenario particularly favorable for evolution of a carotenoid-based ornamentation that de- 
pends on incorporation of environmentally contingent inputs into complex morphological structures.

Our comparisons of feather structure among ornamental parts, individuals, and populations provide additional insights into the evolutionary consequences of genetic assimilation. The finding that pronounced differences in feather shape across ornamental parts are statistically indistinguishable from the patterns induced by dietary carotenoids (fig. $5 a$ corresponding to scenario in fig. $2 a$ ) suggests that time of exposure to and amount of dietary carotenoids (rather than evolved specificity) have a major effect on within-individual variation in feather shape. These results are corroborated by marked differences in the proportion of dietary and derived carotenoids across ornamental parts (table A1), differences in the sequence of molt (and thus carotenoid uptake) across ornamental parts (Badyaev and Vleck 2007), and the fact that widespread dietary carotenoids contributed the most to shape differentiation among body parts (fig. $5 a$ ). Indeed, dietary compounds (e.g., lutein and $\beta$-carotene in finches, adonirubin and cathanaxanthin in spoonbills) contributed up to $10 \%-30 \%$ of variation in the early growing upper part of the feather (landmarks 8 and 7; fig. A1). Early developmental changes in feathers (e.g., determination of barb diameter) covaried with exposure to carotenoids (fig. 7; Landeen and Badyaev 2012). Thus, if dietary carotenoids are present in the feather follicle at earlier developmental stages than derived carotenoids (McGraw 2004), then this could explain their greater contribution to ornamental part related feather reorganization.

Differences among individuals and populations in feather structure were concordant with those exerted by derived carotenoids (fig. $5 b, 5 c$ corresponding to scenario in fig. $2 c$ ). If modification in the structure of a carotenoid-bearing feather evolves by genetic assimilation, then this implies that populations and individuals have evolved differences in metabolic competence or efficiency of carotenoid conversion. Importantly, individuals and populations differed the most in metabolism of biochemically unique carotenoids (fig. $5 e, 5 f$ ). In birds, biochemically unique carotenoids are located at the periphery of long metabolic pathways or occupy distinct biochemical modules (Morrison and Badyaev 2016). Thus, individuals and populations differ the most in either the ability to derive these unique compounds from dietary precursors or the ability to accommodate them into feathers. Both of these explanations suggest that evolved differences in expression of derived carotenoids have a greater genetic basis and lesser context dependence than is often assumed in studies of sexual selection. An important next step for the study of evolution of genetic assimilation is examination of the processes documented in this study in an explicitly historical framework of population divergence (Uller and Helantera 2011; Levis and Pfennig 2016), thereby linking microevolutionary processes of upstream traversing of developmental hierarchies to their macroevolutionary consequences.

\section{Acknowledgments}

We thank R. Duckworth, D. Higginson, and G. Semenov for discussion; F. J. Rohlf for advice with morphometric analyses; and Y. Michalakis and two anonymous reviewers for constructive comments. This study was made possible by the help of dozens of field and laboratory technicians who collected the data and conducted biochemical analyses over the past 15 years. We are especially grateful to $\mathrm{V}$. Belloni, R. Delaney, C. Esposito, V. Farrar, F. Hossein, L. Kennedy, K. King, L. Landeen, and D. Seaman for supervising the teams of lab techs. We thank P. Sweet of the American Museum of Natural History and A. Ramsey of the Reid Park Zoo for providing roseate spoonbill samples, and we also thank C. Davey, S. Davis, and A. Welu for help with feather scanning. This research was supported by grants from the National Science Foundation, the Packard Foundation Fellowship, and the Galileo Fellowship.

\section{Literature Cited}

Badyaev, A. V. 2005. Stress-induced variation in evolution: from behavioural plasticity to genetic assimilation. Proceedings of the Roval Society B 272:877-886.

2014. Epigenetic resolution of the 'curse of complexity' in adaptive evolution of complex traits. Journal of Physiology 592: 2251-2260

Badyaev, A. V., and R. A. Duckworth. 2005. Evolution of plasticity in hormonally-integrated parental tactics. Pages 375-386 in A. Dawson and P. J. Sharp, eds. Functional avian endocrinology. Narosa, New Delhi.

Badyaev, A. V., and K. R. Foresman. 2000. Extreme environmental change and evolution: stress-induced morphological variation is strongly concordant with patterns of evolutionary divergence in shrew mandibles. Proceedings of the Roval Societv B 267:371-377.

Badyaev, A. V., and E. A. Landeen. 2007. Developmental evolution of sexual ornamentation: model and a test of feather growth and pigmentation. Integrative and Comparative Biology 47:221-233.

Badyaev, A. V., E. S. Morrison, V. Belloni, and M. J. Sanderson. 2015. Tradeoff between robustness and elaboration in carotenoid networks produces cycles of avian color diversification. Biology Direct 10:1-22.

Badyaev, A. V., and K. P. Oh. 2008. Environmental induction and phenotypic retention of adaptive maternal effects. BMC Evolutionary Biology 8:e3.

Badyaev, A. V., and C. M. Vleck. 2007. Context-dependent ontogeny of sexual ornamentation: implications for a trade-off between current and future breeding efforts. ournal of Evolutionary Biology 20:1277-1287.

Baldwin, J. M. 1896. A new factor in evolution. American Naturalist 30:441-451. 
1902. Development and evolution. Macmillan, New York.

Bookstein, F. L. 1996. Biometrics, biomathematics and the morphometric synthesis. Bulletin of Mathematical Biology 58:313-365.

Britton, G., G. M. Armitt, S. Y. M. Lau, A. K. Patel, and C. C. Shone. 1982. Carotenoproteins. Pages $237-252$ in G. Britton and T. W. Goodwin, eds. Carotenoid chemistry and biochemistry. Pergamon, Oxford.

Britton, G., S. Liaaen-Jensen, and H. Pfander. 2004. Carotenoid handbook. Birkhäuser, Basel.

Brush, A. H. 1990. Metabolism of carotenoid pigments in birds. FASEB I 4:2969-2977.

Buchecker, R. 1982. A chemist's view of animal carotenoids. Pages 175-193 in G. Britton and T. W. Goodwin, eds. Carotenoid chemistry and biochemistry. Pergamon, Oxford.

Buitrago-Delgado, E., K. Nordin, A. Rao, L. Geary, and C. LaBonne. 2015. Shared regulatory programs suggest retention of blastulastage potential in neural crest cells. Science 348:1332-1335.

Chen, C. F., J. Foley, P. C. Tang, A. Li, T. X. Jiang, P. Wu, R. B. Widelitz, and C. M. Chuong. 2015. Development, regeneration, and evolution of feathers. Annual Review of Animal Biosciences 3:169-195.

Chetverikov, S. S. 1926. On certain aspects of the evolutionary process from the standpoint of modern genetics. Journal of Experimental Biology 2:1-40.

Chuong, C. 1993. The making of a feather: homeoproteins, retinoids and adhesion molecules. Bioessavs 15:513-521.

Cope, E. D. 1887. The origin of the fittest: essays on evolution. Appleton, New York.

Davidson, E. H. 2006. The regulatory genome: gene regulatory networks in development and evolution. Academic Press, San Diego CA.

Dawson, A., and P. J. Sharp. 1998. The role of prolactin in the development of reproductive photorefractoriness and postnuptial molt in the European starling (Sturnus vulgaris). Endocrinology 139: 485-490.

Diggle, P. K., and J. S. Miller. 2013. Developmental plasticity, genetic assimilation, and the evolutionary diversification of sexual expression in Solanum. American Journal of Botany 100:1050-1060.

Dryden, I. L., and K. V. Mardia. 1998. Statistical analysis of shape. Wiley, Chichester.

Efron, B., and R. J. Tibshirani. 1993. An introduction to the bootstrap. Chapman \& Hall, New York.

Ehrenreich, I. M., and D. W. Pfennig. 2015. Genetic assimilation: a review of its potential proximate causes and evolutionary consequences. Annals of Botany 117:769-779.

Emera, D., R. Romero, and G. Wagner. 2012. The evolution of menstruation: a new model for genetic assimilation. Bioessavs 34:2635.

Fox, D. L. 1962. Metabolic fractionation, storage and display of carotenoid pigments by flamingoes. Comparative Biochemistry and Physiology 6:1-40.

Friedman, N. R., K. J. McGraw, and K. E. Omland. 2013. Evolution of carotenoid pigmentation in caciques and meadowlarks (Icteridae): repeated gains of red plumage coloration by carotenoid C4oxygenation. Evolution 68:791-801.

Gaier, K., A. Angerhofer, and H. C. Wolf. 1991. The lowest excited electronic singlet states of all-trans $\beta$-carotene single crystals. Chemical Physics Letters 187:103-109.

García de Blas, E., R. Mateo, J. Viñuela, and C. Alonso-Alvarez. 2011. Identification of carotenoid pigments and their fatty acid esters in an avian integument combining HPLC-DAD and LCMS analyses. Journal of Chromatography 879:341-348.

García de Blas, E., R. Mateo, J. Viñuela, L. Pérez-Rodríguez, and C. Alonso-Alvarez. 2013. Free and esterified carotenoids in ornaments of an avian species: the relationship to color expression and sources of variability. Physiological and Biochemical Zoology 86:483-498.

Gause, G. F. 1940. On the importance of adaptability for natural selection. Journal of General Biology 1:105-120.

Gibson, G., and I. Dworkin. 2004. Uncovering cryptic genetic variation. Nature Reviews 5:681-690.

Goodall, C. 1991. Procrustes methods in the statistical analysis of shape. Journal of the Royal Statistical Society B 53:285-339.

Goodwin, T. W. 1984. Biochemistry of carotenoids. Vol. 2. Animals. Chapman \& Hall, London.

Gossage, C., M. Deyhim, P. B. Moser-Veillon, L. W. Douglas, and T. R. Kramer. 2000. Effect of beta-carotene supplementation and lactation on carotenoid metabolism and mitogenic $\mathrm{T}$ lymphocyte proliferation. American Journal of Clinical Nutrition 71:950-955.

Heil, M., S. Greiner, H. Meimberg, R. Kruger, J.-L. Noyer, G. Heubl, K. E. Linsenmair, and W. Boland. 2004. Evolutionary change from induced to constitutive expression of an indirect plant resistance. Nature 430:205-208.

Hermisson, J., and G. P. Wagner. 2004. The population genetic theory of hidden variation and genetic robustness. Genetics 168: 2271-2284.

Higginson, D. M., V. Belloni, S. N. Davis, E. S. Morrison, J. E. Andrews, and A. V. Badyaev. 2016. Evolution of long-term coloration trends with biochemically unstable ingredients. Proceedings of the Roval Societv B 283:20160403.

Hudon, J. 1991. Unusual carotenoid use by western tanager (Piranga ludoviviana) and its evolutionary implications. Canadian Journal of Zoology 69:2311-2320.

King, N., M. J. Westbrook, S. L. Young, A. Kuo, M. Abedin, J. Chapman, S. Fairclough, et al. 2008. The genome of the choanoflagellate Monosiga brevicollis and the origin of metazoans. Nature 451:783-788.

Klingenberg, C. P., A. V. Badyaev, S. M. Sowry, and N. J. Beckwith. 2001. Inferring developmental modularity from morphological integration: analysis of individual variation and asymmetry in bumblebee wings. American Naturalist 157:11-23.

Klingenberg, C. P., and G. S. McIntyre. 1998. Geometric morphometrics of developmental instability: analyzing patterns of fluctuating asymmetry with Procrustes methods. Evolution 53:1363-1375.

LaFountain, A. M., R. O. Prum, and H. A. Frank. 2015. Diversity, physiology, and evolution of avian plumage carotenoids and the role of carotenoid-protein interactions in plumage color appearance. Archives of Biochemistry and Biophysics 572:201-212.

Landeen, E. A., and A. V. Badyaev. 2012. Developmental integration of feather growth and pigmentation and its implications for the evolution of diet-derived coloration. Journal of Experimental Zoology B 318:59-70.

Levin, M., L. Anavy, A. G. Cole, E. Winter, N. Mostov, S. Khair, N. Senderovich, et al. 2016. The mid-developmental transition and the evolution of animal body plans. Nature 531:637-641.

Levis, N. A., and D. W. Pfennig. 2016. Evaluating 'plasticity-first' evolution in nature: key criteria and empirical approaches. Trends in Ecology and Evolution 2016:563-574.

Lin, S. J., J. Foley, T. X. Jiang, C. Y. Yeh, P. Wu, A. Foley, C. M. Yen, et al. 2013. Topology of feather melanocyte progenitor niche 
allows complex pigment patterns to emerge. Science 340:14421445.

Lucas, A. M., and P. R. Stettenheim. 1972. Avian anatomy: integument. USDA, Washington, DC.

McGraw, K. J. 2004. Colourful songbirds metabolize carotenoids at the integument. Journal of Avian Biology 35:471-476.

. 2006. The mechanics of carotenoid coloration in birds. Pages 177-242 in G. E. Hill and K. J. McGraw, eds. Bird coloration. I. Mechanisms and measurements. Harvard University Press, Cambridge, MA.

Mendes-Pinto, M. M., A. M. LaFountain, M. C. Stoddard, R. O. Prum, H. A. Frank, and B. Robert. 2012. Variation in carotenoid-protein interaction in bird feathers produces novel plumage coloration. Journal of the Roval Societv Interface 9:3338-3350.

Moore, E. F. 1959. The shortest path through a maze. Pages 285-292 in H. Aiken, ed. Proceedings of an International Symposium on the Theory of Switching, April 2-5, 1957, Cambridge, Massachusetts. Harvard University Press, Cambridge, MA.

Morrison, E. S., and A. V. Badyaev. 2016. Structuring evolution: biochemical networks and metabolic diversification in birds. BMC Evolutionary Biology 16:168.

Müller, G. B. 2007. Evo-devo: extending the evolutionary synthesis. Nature Reviews Genetics 8:943-949.

Newman, S. A. 2012. Physico-genetic determinants in the evolution of development. Science 338:217-219.

Newman, S. A., and G. B. Müller. 2000. Epigenetic mechanisms of character origination. Journal of Experimental Zoology 288:304-314.

Olson, S. L. 1970. Specializations of some carotenoid-bearing feathers. Condor 72:424-430.

Osborn, H. F. 1897. Organic selection. Science 15:583-587.

Oster, G., and P. Alberch. 1982. Evolution and bifurcation of developmental programs. Evolution 36:444-459.

Oyama, S. 1988. Stasis, development and heredity. Pages 255-274 in M.-W. Ho and S. W. Fox, eds. Evolutionary processes and metaphors. Wiley, London.

Peter, I., and E. H. Davidson. 2015. Genomic control process: development and evolution. Academic Press, San Diego, CA.

Pigliucci, M., and C. J. Murren. 2003. Genetic assimilation and a possible evolutionary paradox: can macroevolution sometimes be so fast as to pass us by? Evolution 57:1455-1464.

Pigliucci, M., C. J. Murren, and C. D. Schlichting. 2006. Phenotypic plasticity and evolution by genetic assimilation. Journal of Experimental Biology 209:2362-2367.

Prager, M., and S. Andersson. 2010. Convergent evolution of red carotenoid coloration in widowbirds and bishops (Euplectes spp.) Evolution 64:3609-3619.

Price, T., E. Chi, M. Pavelka, and M. Hack. 1991. Population and developmental variation in the feather tip. Evolution 45:518-533.

Price, T. D., A. Qvarnström, and D. E. Irwin. 2003. The role of phenotypic plasticity in driving genetic evolution. Proceedings of the Roval Society B 270:1433-1440.

Prum, R. O., and S. Williamson. 2001. Theory of the growth and evolution of feather shape. Iournal of Experimental Zoology 291:30-57.
Rutherford, S. L., and S. Lindquist. 1998. Hsp90 as a capacitor for morphological evolution. Nature 396:336-343.

Schlichting, C. D., and M. Pigliucci. 1998. Phenotypic evolution: a reaction norm perspective. Sinauer, Sunderland, MA.

Schlichting, C. D., and M. A. Wund. 2014. Phenotypic plasticity and epigenetic marking: an assessment of evidence for genetic accommodation. Evolution 68:656-672.

Schmalhausen, I. I. 1938. Organism as a whole in individual development and history. Academy of Sciences, Leningrad.

Sikkink, K. L., R. M. Reynolds, C. M. Ituarte, W. A. Cresko, and P. C. Phillips. 2014. Rapid evolution of phenotypic plasticity and shifting thresholds of genetic assimilation in the nematode Caenorhabditis remanei. G3 4:1103-1112.

Stebbins, G. L., and D. L. Hartl. 1988. Comparative evolution: latent potentials for anagenetic advance. Proceedings of the National Academy of Sciences of the USA 85:5141-5145.

Stradi, R. 1998. The colour of flight: carotenoids in bird plumage. University of Milan Press, Milan.

Suzuki, Y., and H. F. Nijhout. 2006. Evolution of a polyphenism by genetic accommodation. Science 311:650-652.

Troy, D. M., and A. H. Brush. 1983. Pigments and feather structure of the redpolls, Carduelis flammea and C. hornemanni. Condor 85:443-446.

Tyczkowski, J. K., and P. B. Hamilton. 1986. Lutein as a model dihydroxycarotenoid for the study of pigmentation in chickens. Poultry Science 65:1141-1145.

Uller, T., and H. Helantera. 2011. When are genes 'leaders' or 'followers' in evolution? Trends in Ecology and Evolution 26:435-436.

Waddington, C. H. 1941. Evolution of developmental systems. Nature 147:108-110.

-1953. Genetic assimilation of an acquired character. Evolution 7:119-127.

. 1959. Canalization of development and genetic assimilation of acquired characters. Nature 183:1654-1655.

West-Eberhard, M. J. 2003. Developmental plasticity and evolution. Oxford University Press, Oxford.

2005. Developmental plasticity and the origin of species differences. Proceedings of the National Academy of Sciences of the USA 102:6543-6549.

Wilkins, A. S. 2001. The evolution of developmental pathways. Sinauer, Sunderland, MA.

Wund, M. A., J. A. Baker, B. Clancy, J. L. Golub, and S. A. Foster. 2008. A test of the "flexible stem" model of evolution: ancestral plasticity, genetic accommodation, and morphological divergence in the threespine stickleback radiation. American Naturalist 172: 449-462.

Wyss, A., G. M. Wirtz, W. D. Woggon, R. Brugger, M. Wyss, A. Friedlin, G. Riss, et al. 2001. Expression pattern and localization of $\beta, \beta$-carotene $15,15^{\prime}$-dioxygenase in different tissues. Biochemical Journal 354:521-529.

Associate Editor: Ian Dworkin Editor: Yannis Michalakis 